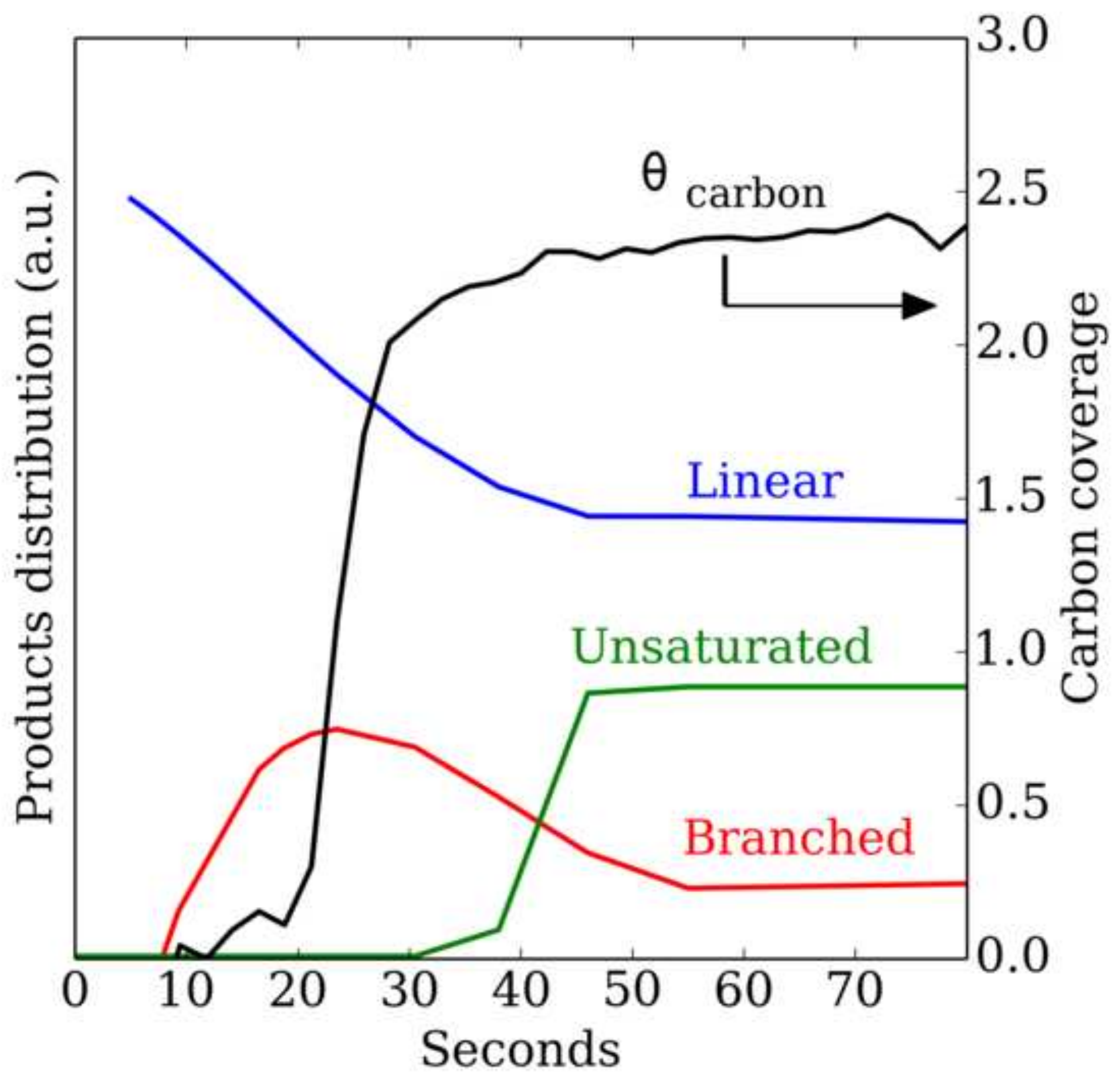




\section{Product Distribution Change in the Early Stages of Carbon Monoxide Hydrogenation over Cobalt Magnesium Fischer-Tropsch Catalyst}

Gérôme Melaet, Walter T. Ralston, Wen-Chi Liu and Gabor A. Somorjait

Department of Chemistry, University of California, Berkeley

Chemical Science Division and Materials Sciences Division, Lawrence

Berkeley National Laboratory, Berkeley

‡ Corresponding Author Email: Somorjai@berkeley.edu 
Keywords: Fischer-Tropsch synthesis, CO hydrogenation, Cobalt, transient experiments, time-resolved, temporal analysis of products

\section{Abstract}

The catalytic hydrogenation of carbon monoxide, known as the FischerTropsch process, is a technologically important, complex multipath reaction which produces long chain hydrocarbons. In order to access the initial kinetics and the mechanism, we developed a reactor that provides information under non-steady state conditions. We tested a CoMgO catalyst and monitored the initial product formation within 2 seconds of exposure to $\mathrm{CO}$ as well as the time dependence of high molecular weight products (in a 60 second window) and found drastic changes in the product selectivity. The probability for forming branched isomers $\left(\mathrm{C}_{4}\right.$ and $\left.\mathrm{C}_{5}\right)$ peaks in the first 25 seconds, and within that time frame no unsaturated products were detected. The subsequent decline (at $\sim 35$ to 40 seconds) of branched isomers coincides with the detection of olefins (from $\mathrm{C}_{2}$ to $\mathrm{C}_{5}$ ) and the change in carbon coverage at the surface of the catalyst. This indicates a change in the reaction pathway.

\section{Introduction}

Continuous growth of the worldwide population is increasing the demands in energy leading to the depletion of fossil sources. Crude oil is the primary source for fuel but also for fine chemical production and the plastics industry. 
Besides the use of conventional alternative sources of energy such as wind, solar, or nuclear power, the synthesis of higher value hydrocarbons from carbon monoxide and hydrogen is an attractive solution. This solution, known as Fischer-Tropsch synthesis, has been around since the early 1900s. Fischer-Tropsch is a perfect example of the greatest challenge heterogeneous catalysis has to face: control of selectivity [1,2]. The major drawback of this synthesis is its vast product distribution and the lack of precise control on the selectivity [3-8]. Subsequent refining processes (hydrocracking, isomerization, reforming, alkylation, etc...) are needed in order to obtain the desired product. Our approach to this problem, until now, has been to use model nanoparticle catalysts in which the synthesis of both the support and the active phase is well controlled [9-11], and to study these model catalysts under high pressure steady state conditions.

Despites their prevalence, steady state experiments are severely limited in that they provide only global kinetics parameters and little to no information on individual reactions steps. Chemical Transient Kinetic experiments and Temporal Analysis of Products are among the most helpful techniques to evaluate the intrinsic kinetic coefficients and give information on the different steps of the reaction [12-17].

In the present paper, we are introducing our findings regarding the initial changes in the product distribution of the FT synthesis. This was made possible by the combination of a new reactor enabling Chemical Transient Kinetics (CTK) experiments with Temporal Analysis of the Products. 


\section{Material and Methods}

\subsection{Catalyst Preparation}

Cobalt magnesium oxide catalyst was prepared according to literature using the oxalate route as described elsewhere [14]. Briefly, a bimetallic solution of cobalt nitrate $\left(\mathrm{Co}\left(\mathrm{NO}_{3}\right)_{2}\right.$, Sigma Aldrich) and magnesium nitrate $\left(\mathrm{Mg}\left(\mathrm{NO}_{3}\right)_{2}\right.$, Sigma Aldrich) is prepared in acetone with an atomic ratio of 10 to

1. The mixed Co-Mg oxalate was obtained by addition of oxalic acid also dissolved in acetone. The resulting precipitate was centrifuged, washed several times and dried overnight. Subsequently, the dried substance was crushed and sieved to obtain the catalyst precursor $(150 \mu \mathrm{m}<$ particulates size $<250 \mu \mathrm{m})$. The final catalyst was obtained by thermal decomposition of the oxalate in hydrogen ( $10 \%$ in argon) at $400^{\circ} \mathrm{C}$.

\subsection{Catalyst Characterization}

Physical and metallic surface areas of the catalyst were measured using a Micromeritics ASAP 2020. In both cases the oxalate was decomposed in situ with pure hydrogen at $3^{\circ} \mathrm{C} / \mathrm{min}$ up to $400^{\circ} \mathrm{C}$. Prior to the measurement, the $\mathrm{CoMgO}$ sample was evacuated at $150^{\circ} \mathrm{C}$ for 2 hours. The physisorption measurements were taken using nitrogen as the probe at $196^{\circ} \mathrm{C}$, and the surface area was extrapolated using the BET expression. The metallic surface area was obtained using hydrogen as a probe molecule. The hydrogen chemisorption was carried out at $35^{\circ} \mathrm{C}$ and the surface area was extrapolated from the isotherm. Scanning Electron Microscopy (SEM) and Energy Dispersive Spectroscopy mapping (EDS) was acquired using an 
Analytical Field Emission Zeiss Gemini Ultra-55 (at $20 \mathrm{KeV}$ ). X-ray

Photoelectron Spectroscopy experiments were performed in a PHI ESCA instrument at a base pressure of $7.510^{-9}$ mbar using a non-monochromatic $\mathrm{Al}$ Ka radiation. Photoelectron core-level spectra were acquired using a hemispherical analyzer at a pass-energy of $35.75 \mathrm{eV}$ with a $0.05 \mathrm{eV}$ energy step. The spectra were analyzed using the public software package CasaXPS.

\subsection{Catalytic Testing}

Transient experiments or Chemical Transient Kinetics experiments were conducted in a home built system described in a previous work [10]. The system is equipped with two independent plug-flow circuits connected to a small volume reactor via a 4-way valve (see Figure 1 for a schematic).

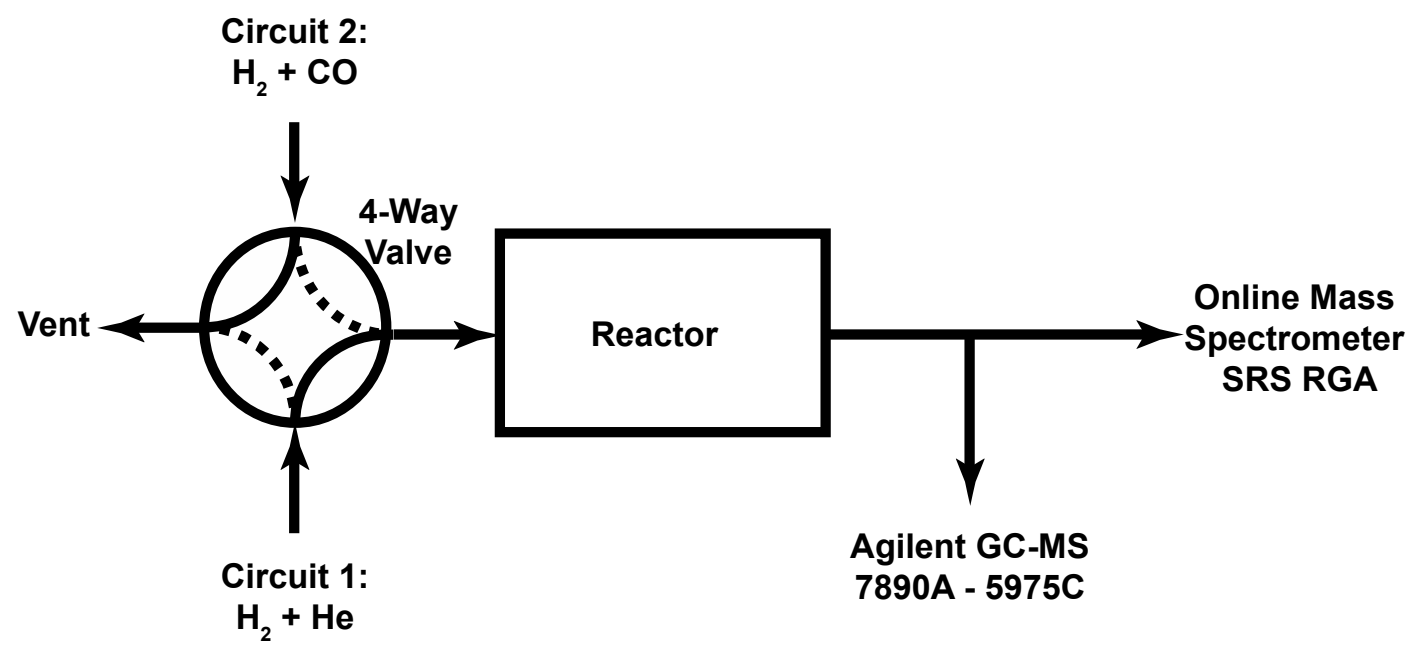

Figure 1: Schematic of the Chemical Transient Kinetics system used for this study: the 4-way valve allows for quick change of gaseous composition while the outlet is monitored online by a mass spectrometer (SRS RGA). The use of a secondary analytical system, here an Agilent GCMS, allows for Temporal Analysis of Products during the transient period. 
This 4-way valve enables a fast and reproducible switch of the gaseous composition directed to the catalysts by switching from one circuit to the other. The outlet of the gas reactor is monitored online using a mass spectrometer (RGA, Stanford Research System) equipped with a differentially pumped capillary and valve inlet system (Pfeiffer GES-010). This inlet system allows for a fast sampling rate $(\max =0.5 \mathrm{~s})$ while keeping the ionization chamber under molecular flow conditions providing a means for quantitative measurements. In practice, the number of mass/charge ratios monitored using the multiple ion detection mode limits the time resolution. In the present work, we have decided to follow $14 \mathrm{~m} / \mathrm{z}$ ratios giving an optimum time resolution of 2.2s. In addition to the online mass spectrometer, small aliquots of the gas outlet are analyzed by Gas Chromatography combined with a Mass Spectrometer (Agilent GC-MS). The volume sampled from the outlet corresponds to $\sim 1.3 \mathrm{~mL}$, which given the mass flow rate, is equivalent to a time resolution of 2 s allowing for a Temporal Analysis of Products (TAP).

Catalytic tests were operated at constant temperature of $230^{\circ} \mathrm{C}$ with a ratio of $\mathrm{H}_{2}$ to $\mathrm{CO}$ equal to $3: 1$. Prior to the testing, the oxalate precursor is decomposed in situ as described earlier.

\section{Results and Discussion}

The physico-chemical characteristics of the catalyst are presented in Table 1. According to the metallic surface area obtained from $\mathrm{H}_{2}$ chemisorption, we evaluated the cobalt particles average diameter to be 38nm. SEM and EDS mapping evidence a homogeneous distribution of Co 
and $\mathrm{Mg}$ within the catalytic grains (see Figure 2a). Moreover the post-mortem sample presents an identical homogeneous distribution of both $\mathrm{Mg}$ and $\mathrm{Co}$ in the grains (Figure 2b). Nevertheless, surface segregation cannot be ruled out due to the low spatial resolution of the EDS mapping. Finally, the EDS analysis of the samples confirms the atomic percentage to be $89 \%$ Co and $11 \% \mathrm{Mg}$.

\begin{tabular}{|l|c|}
\hline CoMgO & \\
\hline Nominal Composition & $10: 1$ Co:Mg \\
\hline Particle size $(\mathrm{nm})$ & 38 \\
\hline BET $\left(\mathrm{m}^{2} / \mathrm{g}\right)$ & 89.5 \\
\hline Metallic surface $\left(\mathrm{m}^{2} / \mathrm{g}\right.$ of catalyst $)$ & 4.7 \\
\hline
\end{tabular}

Table 1: Description of the CoMgO catalyst after decomposition of the oxalate precursor using a temperature programmed decomposition under $\mathrm{H}_{2}(10 \%$ in $\mathrm{Ar})$ up to $400^{\circ} \mathrm{C}$ at a rate of $6^{\circ} \mathrm{C} . \mathrm{min}^{-1}$

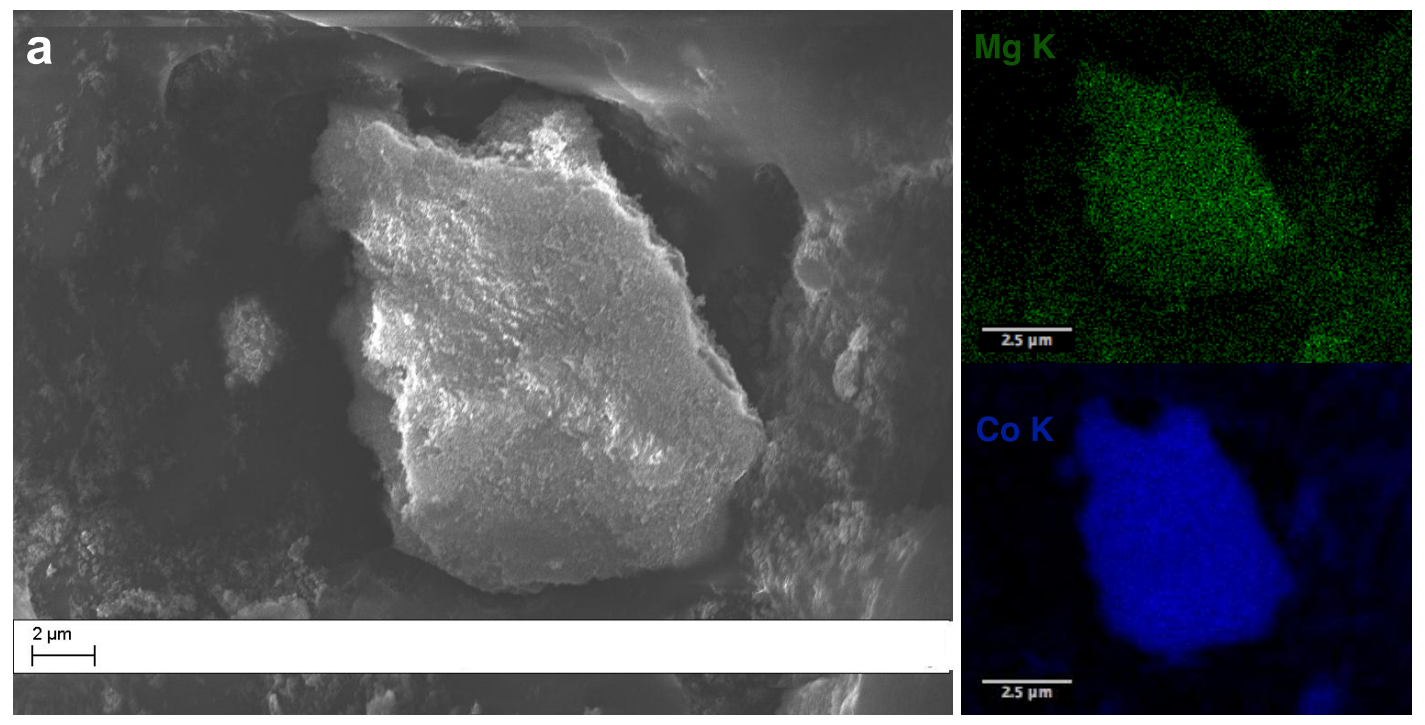




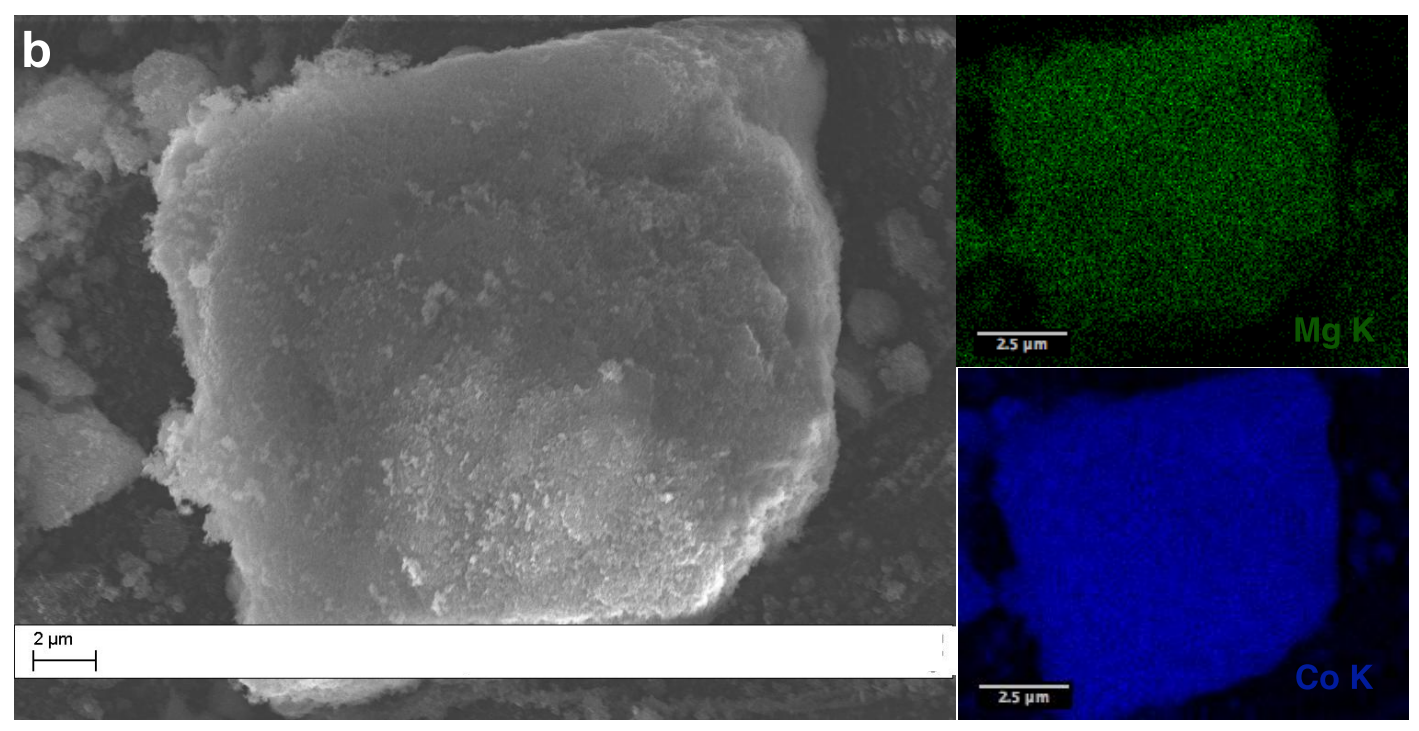

Figure 2: SEM image and EDS mapping of passivated samples. a) Pristine and b) spent.

The turnover frequency was evaluated at steady state based on the total cobalt metallic surface area and CO conversion. It was found that the CO conversion at steady state is $32 \%$ giving a TOF of $0.066 \mathrm{~s}^{-1}$. In addition, the product distribution at steady state shows high selectivity towards methane and large chain hydrocarbons $\left(\mathrm{C}_{5+}\right)$ and is comparable to the selectivity of other cobalt-based catalysts.

Time transient data obtained by the online MS are presented in Table 2.

\begin{tabular}{|l|c|}
\hline & Transient data \\
\hline Products & Delay Time (s) \\
\hline $\mathrm{CO} \& \mathrm{CO}_{2}$ & 21 \\
\hline $\mathrm{CH}_{4}$ & 9 \\
\hline $\mathrm{C}_{2 \mathrm{~s}}$ & 12 \\
\hline $\mathrm{C}_{3 \mathrm{~s}}$ & 14 \\
\hline
\end{tabular}

Table 2: Delay times obtained during the transient period or surface build up. The times are given in seconds and evaluated from the time of switch using Ar as an internal standard. 
Data gathered both from the online MS and from the aliquots sampled during the transient period (see supporting information) show that the sequence in which the carbon chains appeared is directly related to the number of carbon atoms in the hydrocarbon chain. In fact, the products appear in the following order: $\mathrm{C}_{2 \mathrm{~s}}-\mathrm{C}_{3 \mathrm{~s}}-\mathrm{C}_{4 \mathrm{~s}}-\mathrm{C}_{5 \mathrm{~s}}-\mathrm{C}_{6 \mathrm{~s}}$. Moreover, it should be pointed out that these hydrocarbons appear within 40s of the build-up (Figure 3 ) and that $\mathrm{C}_{6}$ is the longest hydrocarbon chain observed.

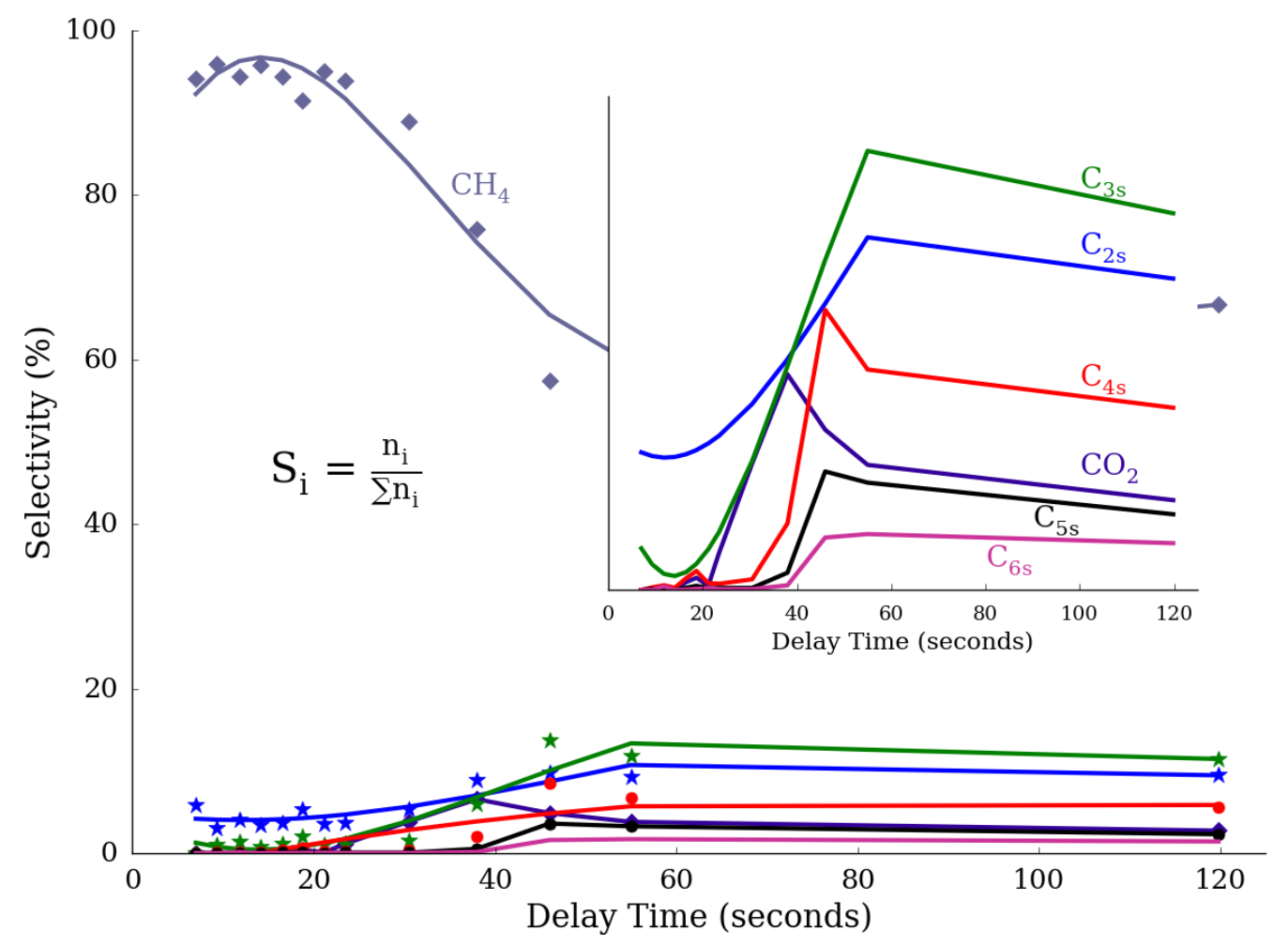

Figure 3: Selectivity as a function of time for different products obtained from aliquots at the GCMS during the transient period of Fischer-Tropsch synthesis on CoMgO. Conditions: atmospheric pressure, $230^{\circ} \mathrm{C}$ and $\mathrm{H}_{2}: \mathrm{CO}=3: 1$.

The use of GC-MS allows for the differentiation of isomers and saturated/unsaturated molecules. According to our data we have evidence of a change in the product distribution in the early stage of the FT synthesis. Initially, branched and linear molecules are produced simultaneously, whereas it takes about 40 seconds to detect the first unsaturated product (see 
Figure 4a). We have evaluated the probability for the catalyst to produce a branched molecule versus its linear equivalent (Figure 4b). It appears that the production of branched isomers peaks in the first 25 seconds during the buildup of the surface before decreasing and reaching a steady production rate around 50 seconds. It is interesting to notice that this decline in branched molecules corresponds to the onset of the unsaturated products (Figure 4).
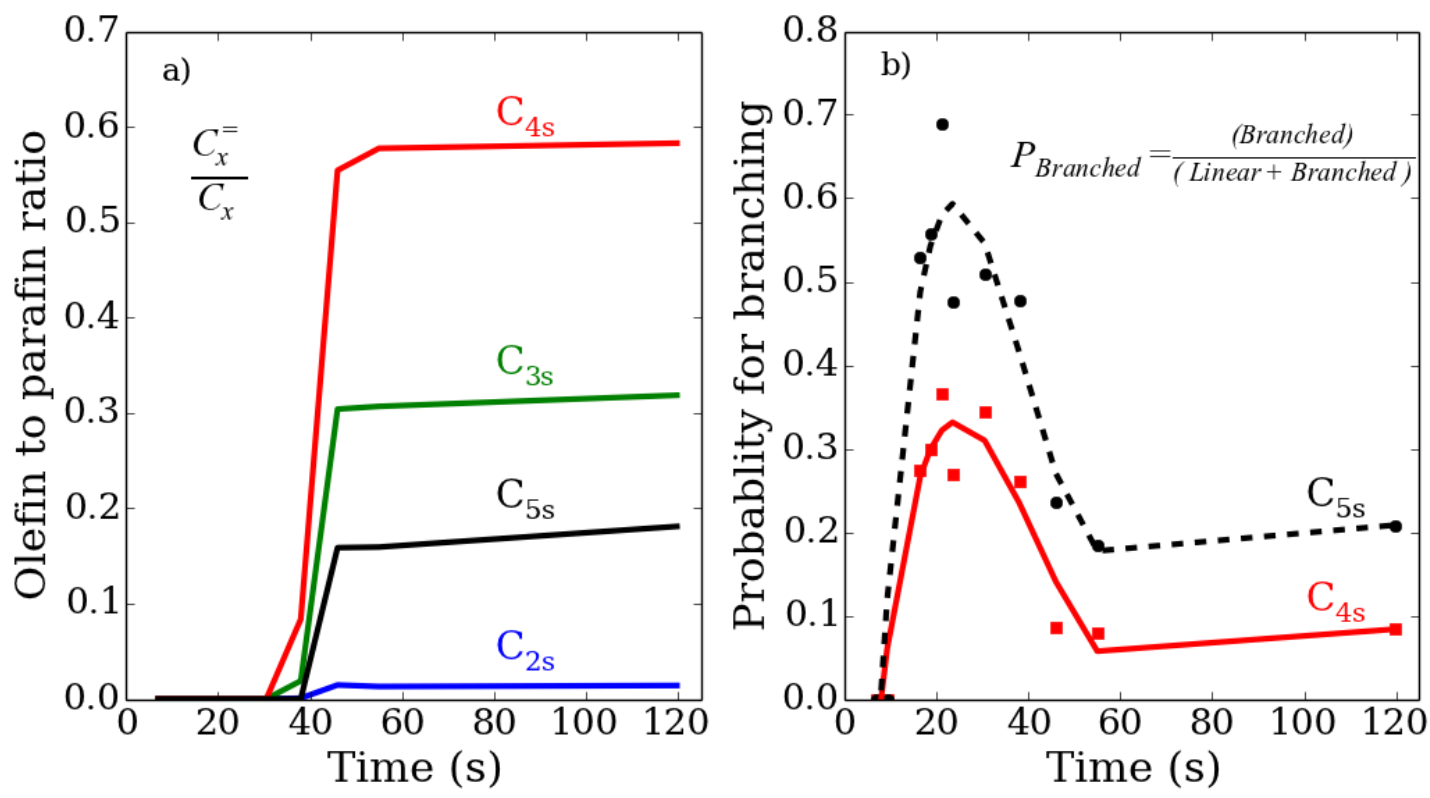

Figure 4: Changes in the selectivity in the Fischer-Tropsch products during the transient period highlighted by: a) the change in the olefin to paraffin ratio and b) the change in the probability to produce branched molecules.

Moreover, the carbon coverage at the surface of the catalyst was evaluated over time using the following expression:

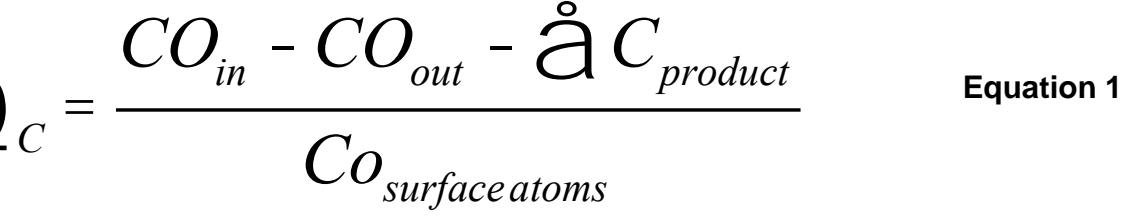

Where the $\mathrm{CO}_{\text {in }}$ and $\mathrm{CO}_{\text {out }}$ were evaluated from the online $\mathrm{MS}$ data and the carbon in the different products were evaluated using the GC-MS data. 
The results are plotted in Figure 5 against the data obtained for the linear, unsaturated and branched molecules.

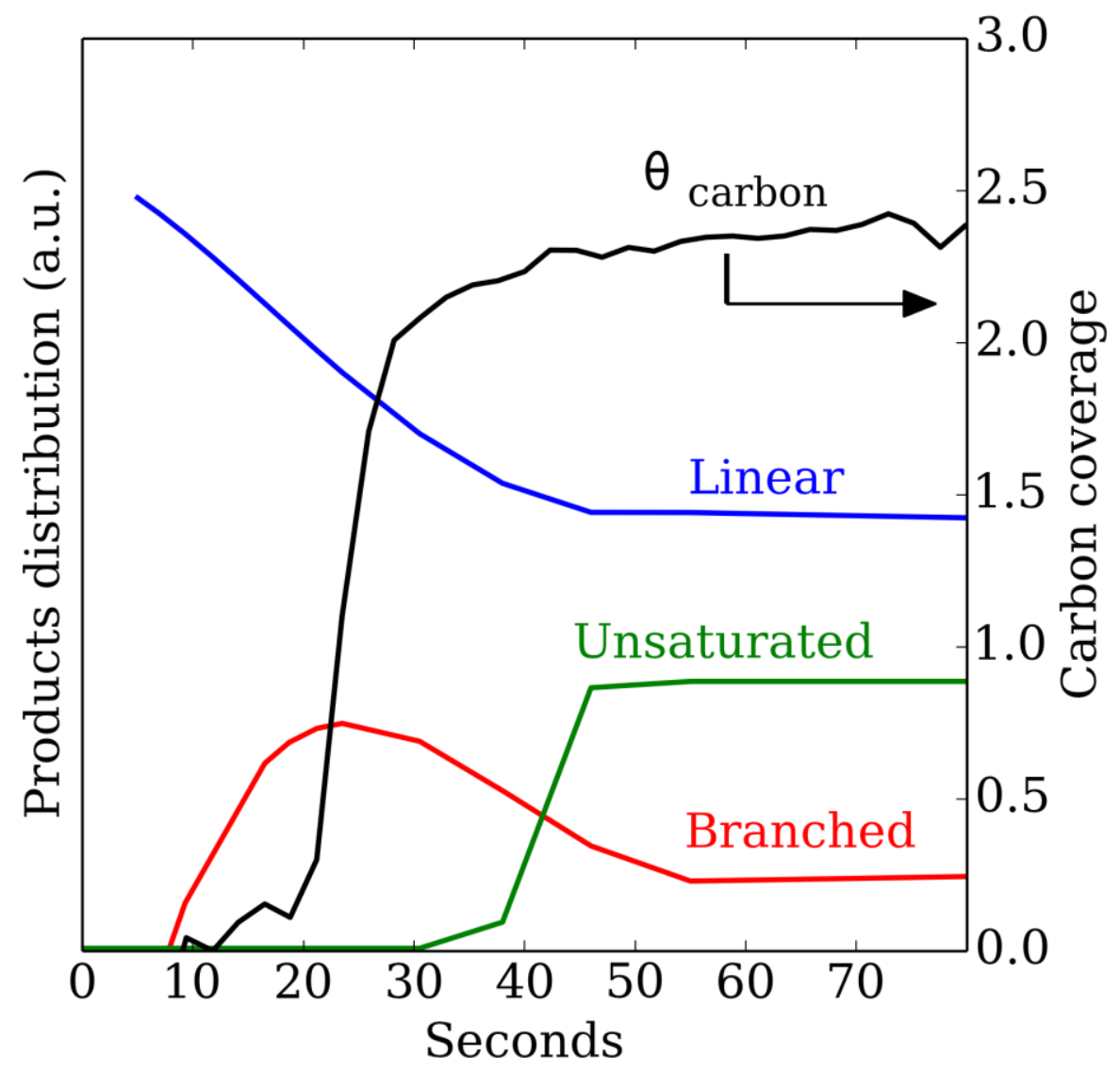

Figure 5: Product distribution as a function of time and carbon coverage on CoMgO catalysts at $230^{\circ} \mathrm{C}$ and $40 \mathrm{sccm}\left(\mathrm{H}_{2}: \mathrm{CO}=3: 1\right)$

Within the first seconds all the carbon monoxide put in the inlet is converted to product with a selectivity of $95 \%$ to methane. The appearance of the branched product coincides with the onset of carbon coverage at the cobalt surface. As the coverage reach $\sim 0.80$, the product distribution starts to change in favor of unsaturated molecules. Meanwhile, the methane selectivity decreases from its initial value to $65 \%$. What seems to emerge from this analysis is the change in the pathway from 1-branched to 1-alkenes linked with the increase of carbon coverage, and is indicative of a sterical hinderance at the surface. 
Once steady state is reached, the carbon coverage reaches a constant value of 2.5 carbon per cobalt surface atoms. This value is seemingly high but is a lower limit, as the oxygen and hydrogen coverage are complex to evaluate and probably decrease the amount of available surface sites.

The number of carbon atoms per cobalt atoms at steady state can be easily conceived if one considers the polymerization process occurring during the Fischer-Tropsch synthesis. Based on the partial pressure of the products in the outlet flow, we have determined the most abundant carbon chain at the surface of the catalysts. According to the data presented in Figure 6, we estimated the most abundant hydrocarbon chain at the surface to be a three carbons chain.

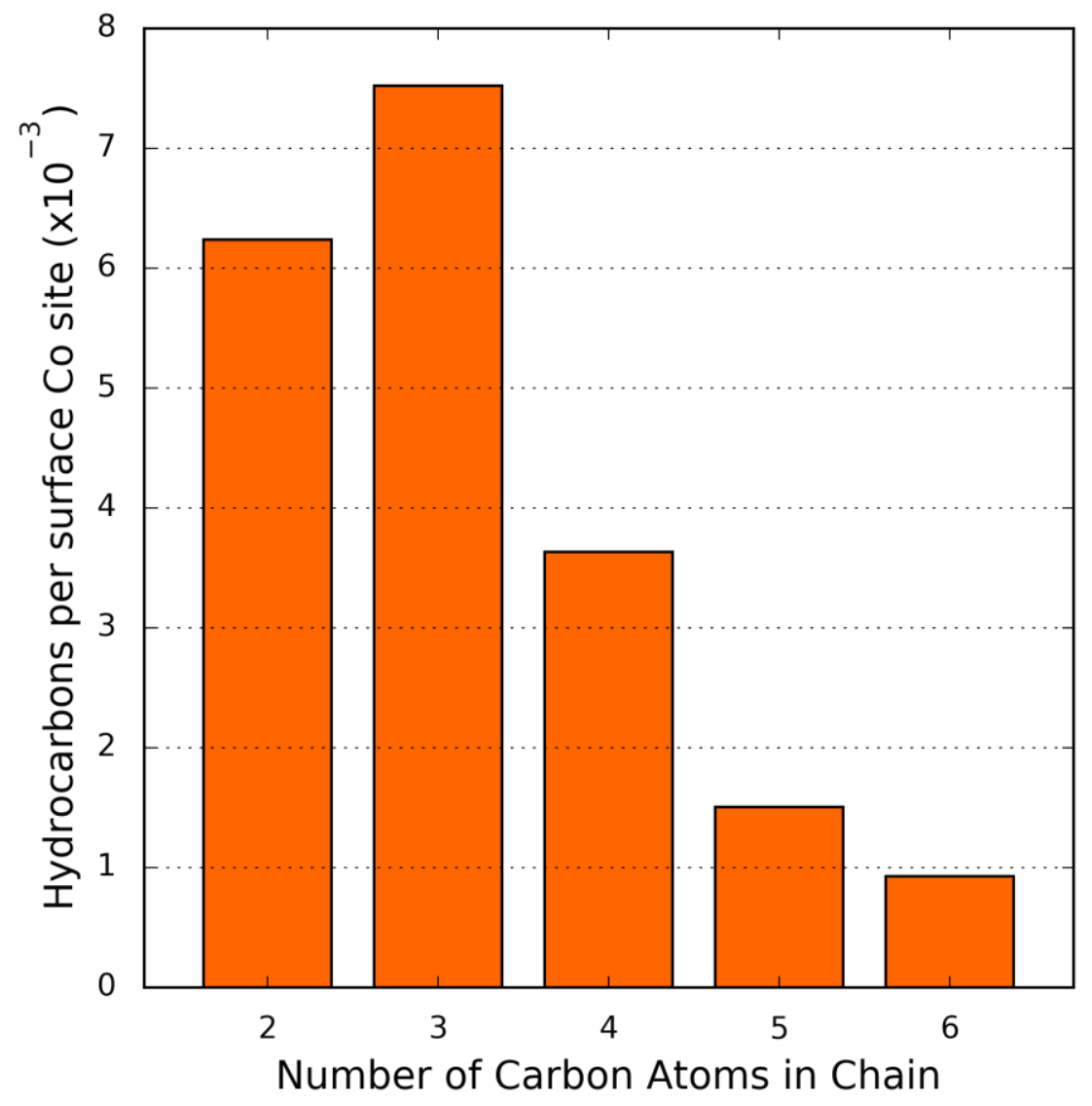

Figure 6: Distribution of hydrocarbons with a chain length of $\mathrm{N}$ carbon atoms per cobalt surface sites. Methane was purposely left aside as it is the main product and occupies a large portion of the surface. $\mathrm{CO}_{2}$ was only found in trace amounts. 
Nevertheless, the possibility of carbon dilution in the cobalt subsurface or even the formation of cobalt carbide cannot be ruled out at this point. In fact, pristine samples (after decomposition of the oxalate) and post-mortem samples have been investigated using lab XPS. Figure S3 shows both the $\mathrm{C} 1 \mathrm{~s}$ and Co $2 \mathrm{p}$ regions (a and $\mathrm{b}$, respectively). The carbon 1s envelop shows mainly a peak centered around $285 \mathrm{eV}$ indicating the presence of adventitious carbon. We attributed the presence of these carbon species to the fact that the samples had to be transferred in air from our reactor to the XPS system. No carbide could be identified and there is no obvious difference between the spectra of both samples.

The Co $2 \mathrm{p}_{3 / 2}$ B.E. at $\sim 780 \mathrm{eV}$ indicates the presence of oxidized cobalt, which is consistent with the fact that our samples were passivated in $\mathrm{O}_{2}$ prior to being exposed to air. Once again, no obvious evidence of a cobalt carbide, or carbon dilution in the cobalt particles, could be highlighted. It should be noted that the used sample was exposed to a back transient, i.e. the flow was switched from $\mathrm{H}_{2} / \mathrm{CO}$ to $\mathrm{H}_{2} / \mathrm{He}$ and a large peak of methane was observed indicating that carbon left at the surface would have reacted with the hydrogen. In order to confirm the incorporation of carbon in the cobalt particles, in situ measurement such as Near-Edge X-ray Absorption Fine Structure (NEXAFS) spectroscopy or Ambient Pressure XPS should be run.

\section{Conclusions}

Using a Chemical Transient Kinetics experiment combined with Temporal Analysis of Products with a time resolution of $\sim 2 \mathrm{~s}$., we have demonstrated 
that the Fischer-Tropsch synthesis undergoes drastic product distribution changes in the very first seconds of the reaction. Our new operando system showed that even at low pressure (1atm versus 20 bar in industrial condition) longer hydrocarbon chains such as $\mathrm{C}_{6}$ can be formed. Moreover, we have evidenced that both linear and branched molecules are produced simultaneously. The appearance of unsaturated molecules seems to be linked to the decline in branching probability. Using the data obtained by online mass spectrometry and the temporal analysis of products by GC-MS, we have linked the change in product distribution to the change in carbon coverage. As the build-up of the surface continues, steric effects cause the catalyst to evolve toward other preferential routes.

Catalytic materials, such as the one use in the framework of this study, display heterogeneities in terms of composition, structure, and thus reactivity of active sites. To achieve the $100 \%$ selectivity milestone in heterogeneous catalysis, it is paramount to understand the interaction between the gas-solid interface and how the structure influences the reaction as well as how the gaseous composition restructures and shapes the catalyst. In the near future, we are planning to use a similar technique and apply this CTK/TAP to a model catalyst made of well-controlled cobalt nanoparticles. We hope to provide new insights on the structural importance of the cobalt on the product distribution. Similarly, the use of CTK/TAP applied to other reactions such as tandem catalysis (i.e. detection of intermediate reactant to secondary reaction) or bi-functional catalysis (i.e. metal assisted acid catalysis for isomerization reactions) will allow for shedding light on the mechanism of these reactions. 
In parallel, the use of this method combined with in situ characterization such as sum frequency generation spectroscopy, x-ray adsorption, x-ray emission, and photoelectron spectroscopies [18] will help define the key factors at an atomic level which influence the kinetics of reactions as well as the restructuring occurring in nanomaterials often used in heterogeneous catalysis.

\section{Acknowledgment}

The present paper was submitted in honor of Dr. Jens Rostrup-Nielsen. The authors want to thank the Molecular Foundry of the Lawrence National Laboratory for the help in the SEM and EDS (Proposal \#3806). This work was supported by the U.S. Department of Energy, Office of Science, Office of Basic Energy Sciences, Chemical Sciences, Geosciences, and Biosciences Division under Contract DE-AC02-05CH11231

\section{References}

[1] G.A. Somorjai, J.Y. Park, Angew. Chem. Int. Ed. 47 (2008) 9212-28

[2] G.A. Somorjai, Y. Li, Proc Natl Acad Sci U S A 108 (2011) 917-24

[3] G.L. Bezemer, J.H. Bitter, H.P. Kuipers, H. Oosterbeek, J.E. Holewijn, X. Xu, F. Kapteijn, A.J. van Dillen, K.P. de Jong, J. Am. Chem. Soc. 128 (2006) $3956-64$

[4] J.P. den Breejen, P.B. Radstake, G.L. Bezemer, J.H. Bitter, V. Frøseth, A. Holmen, K.P. de Jong, J. Am. Chem. Soc. 131 (2009) 7197-203 
[5] G. Prieto, S. Beijer, M.L. Smith, M. He, Y. Au, Z. Wang, D.A. Bruce, K.P. de Jong, J.J. Spivey, P.E. de Jongh, Angew. Chem. Int. Ed. 53 (2014) 6397401

[6] E. van Steen, M. Claeys, Chemical Engineering \& Technology 31 (2008) $655-666$

[7] D. Tristantini, S. Lögdberg, B. Gevert, Borg, A. Holmen, New Journal 88 (2007) 643-649

[8] Borg, P.D. Dietzel, A.I. Spjelkavik, E.Z. Tveten, J.C. Walmsley, S. Diplas, S. Eri, A. Holmen, E. Rytter, J. Catal. 259 (2008) 161-164

[9] G. Melaet, W.T. Ralston, C.S. Li, S. Alayoglu, K. An, N. Musselwhite, B. Kalkan, G.A. Somorjai, J. Am. Chem. Soc. 136 (2014) 2260-3

[10] G. Melaet, W.T. Ralston, W.-C. Liu, G.A. Somorjai, J. Phys. Chem. C 118 (2014) 26921-26925

[11] G. Melaet, A.E. Lindeman, G.A. Somorjai, Top. Catal. 57 (2014) 500-507 [12] A. Frennet, C. Hubert, J. Mol. Catal. A: Chem. 163 (2000) 163-188

[13] E. Vesselli, J. Schweicher, A. Bundhoo, A. Frennet, N. Kruse, J. Phys. Chem. C 115 (2010) 1255-1260

[14] J. Schweicher, A. Bundhoo, N. Kruse, J. Am. Chem. Soc. 134 (2012) $16135-8$

[15] S. Vada, A. Hoff, E. Ådnanes, D. Schanke, A. Holmen, Top. Catal. 2 (1995) 155-162

[16] V. Frøseth, S. Storsæter, Borg, E.A. Blekkan, M. Rønning, A. Holmen, Appl. Catal., A 289 (2005) 10-15

[17] S.L. Shannon, J.G. Goodwin Jr, Chem. Rev. 95 (1995) 677-695 
[18] H.-L. Han, G. Melaet, S. Alayoglu, G.A. Somorjai, ChemCatChem 7 (2015) 3265-3638 